\section{Revista Brasileira de Administração Científica \\ Brazilian Journal of Scientific Administration}

Jan a Abr 2018 - v.9 - n.1

\title{
Gerenciamento de risco em projetos de consultoria
}

Tendo em vista que quaisquer organizações não estão livres de cometerem falhas e correr riscos, o presente trabalho traz a integração de alguns métodos de Gerenciamento de Risco que foram capazes de identificar, analisar e avaliar riscos dentro dos projetos de uma empresa de prestação de serviços. Apesar do gerenciamento de risco ser um processo capaz de ser empregado em diversos tipos de organizações e situações, muitas organizações não tem o conhecimento de suas técnicas. Assim, a presente pesquisa tem como objetivo identificar as falhas capazes de trazer consequências negativas para os projetos da empresa, além de identificar as falhas nos projetos, a pesquisa também identificou as causas de cada uma das falhas, bem como, para cada falha identificada foi sugerida ações capazes de mitiga-las. Entre os métodos utilizados estão as técnicas de Brainstorming, Análise de Causa Raiz, Diagrama de Causa e Efeito e a Análise de Modos de Falhas. Como resultados alcançados, foi verificado que o trabalho está dentro dos passos da norma ISO 31000 que conta com identificação, análise e avaliação de riscos. Ademais, este trabalho também conta coma determinação de ações corretivas. $\mathrm{O}$ artigo contribui como uma pesquisa empírica no ramo de Gerenciamento de Risco voltado a projetos de prestação de serviços.

Palavras-chave: Gerenciamento de Risco; Prestação de Serviço; Projetos.

\section{Risk management in business projects}

Given that any organization is not free to commit failures and take risks, the present work brings the integration of some methods of Risk Management that were able to identify, analyze and evaluate risks within the projects of a service company. Although risk management is a process that can be employed in many types of organizations and situations, many organizations are not aware of their techniques. Thus, the present research aims to identify the flaws that can have negative consequences for the company's projects, in addition to identifying the flaws in the projects, the research also identified the causes of each failure, as well as, for each identified failure actions to mitigate them. Among the methods used are the techniques of Brainstorming, Root Cause Analysis, Cause and Effect Diagram and Failure Mode Analysis. As results achieved, it was verified that the work is within the steps of the ISO 31000 standard that counts with identification, analysis and evaluation of risks. In addition, this work also counts on the determination of corrective actions. The article contributes as an empirical research in the field of Risk Management focused on service projects.

Keywords: Risk management; Provision of Service; Projects.

Topic: Teoria Geral da Administração

Reviewed anonymously in the process of blind peer.
Received: 11/01/2019

Approved: 21/04/2019
Diego Pereira Ribeiro (iD)

Universidade Federal Fluminense, Brasil http://lattes.cnpq.br/0200488166833935 http://orcid.org/0000-0002-7828-7967 dyegopr@yahoo.com.br

Ualison Rébula Oliveira (id)

Universidade Federal Fluminense, Brasil http://lattes.cnpq.br/6460931837390456 http://orcid.org/0000-0002-8097-4889 ualison.oliveira@gmail.com

\section{Referencing this:}

RIBEIRO, D. P.; OLIVEIRA, U. R.. Gerenciamento de risco em projetos de consultoria. Revista Brasileira de Administração Científica, v.9, n.1, p.14-31, 2018. DOI: http://doi.org/10.6008/CBPC2179$684 X .2018 .001 .0002$ 


\section{INTRODUÇÃO}

No atual cenário dos negócios, existe uma grande pressão ao rápido desenvolvimento tecnológico e às mudanças contínuas nas demandas dos clientes. Neste contexto, as empresas estão a enfrentar uma concorrência vigorosa em termos de qualidade, custos e tempo. Para lidar com essas tendências, as demandas exigem maior flexibilidade e padrões de qualidade, mas essas exigências geram a necessidade de altos níveis de confiabilidade do sistema, que inclui os elementos humanos, equipamentos, materiais e outros processos de valor agregado, além disso, as organizações ainda enfrentam eventos adversos e contratempos que tornam o sistema pouco confiável. Como consequência, pode haver dificuldades na continuidade do trabalho, diminuição da produtividade, aumento de produtos defeituosos, retrabalho, atraso na entrega, perda humana, perda financeira entre outros (ARIFUL et al., 2006).

As empresas precisam ser capazes de lidar com as capacidades internas para responder a esses eventos que podem afetar sua capacidade. Em todo o mundo as empresas parecem cometer os mesmos erros continuamente nos processos de desenvolvimento de produtos e de produção industrial, portanto, as empresas estão em busca de abordagens rápidas que respondam a essas questões sem comprometer a qualidade dos produtos, processos e serviços (REID et al., 2012).

As complexidades dos negócios modernos exigem abordagens mais sofisticada, então os líderes precisam estabelecer políticas para resolução de problemas e técnicas de análises estruturadas. A alternativa é continuar a assumir que os esforços existentes produzirão resultados diferentes, mas, assumir que se pode trabalhar em um problema sem um método para avaliação crítica é uma política condenada a falhar (DOGGETT, 2005).

O sucesso de um projeto é ponto crítico para o desempenho do negócio. Mesmo assim, muitos projetos ainda sofrem atrasos e até mesmo fracassos. Com as mudanças rápidas e dinâmicas no cenário econômico atual, não é suficiente ter apenas um bom plano de projeto, ou um acompanhamento adequado de sistema de controle, as organizações precisam estar preparadas para os riscos e estarem prontas para fazer algo a respeito, no entanto, os métodos de gestão de risco que foram desenvolvidos para melhorar o sucesso dos projetos são pouco utilizados (RAZ et al., 2002).

Segundo Raz et al. (2002) os riscos de um projeto podem vir dos próprios processos da organização, que podem ser caracterizados por incertezas, complexidade e urgência, por falta de recursos ou outras restrições, tais como habilidades ou política. Enquanto ninguém pode evitar riscos nos projetos (assim como ninguém pode evitar catástrofes naturais ou incêndios), certamente pode se preparar para os riscos, adicionando atividades de gestão de risco aos planos de projeto e implantação de mecanismos que irão proteger a organização quando algo sair errado.

Ariful et al. (2006) explica que a gestão de riscos se refere ao processo de redução dos riscos. Os métodos baseados em risco fornecem várias medidas qualitativas e quantitativas que podem apoiar a tomada de decisões. O principal objetivo é manter os riscos a um nível aceitável, mantendo os riscos toleráveis e seguindo um programa para transferir riscos inaceitáveis para um nível aceitável. Segundo 
Mulisani et al. (2014), a gestão de risco é um processo contínuo e disciplinado de planejamento, identificação, análise, desenvolvimento de respostas, monitoramento e controle aos riscos, que pode ser aplicado em projetos com diferentes tipos de complexidades.

A fim de identificar, analisar, avaliar e propor ações mitigadoras aos riscos nos projetos de uma empresa de prestação de serviço, o presente trabalho procura responder os seguintes questionamentos: Quais são as possíveis falhas capazes de trazer consequências negativas para os projetos da empresa? O que pode causar estas falhas? Como reduzir e evitar estas falhas nos projetos?.

A presente pesquisa trata de um estudo de caso empírico em uma empresa de prestação de serviços de consultoria de negócios. O objetivo da empresa é aumentar o desempenho de seus clientes por meio de projetos de consultoria. Tais projetos envolvem análises financeiras, análises operacionais e formulação de estratégias com a finalidade de aumentar a produtividade e a eficiência do cliente. É preciso entregar os projetos dentro dos prazos e ainda dentro da expectativa dos clientes. Deve-se levar em consideração que um projeto de consultoria nunca é igual a outro projeto realizado anteriormente, pois os clientes são diferentes, possuem ramos de atividades diferentes e estão em cenários diferentes. Sendo assim, cada projeto de consultoria é único e específico para cada cliente, os serviços prestados são sempre diferenciados. Segundo Salavati et al. (2016), clientes que exigem serviços específicos aumentam o potencial para a existência de risco.

A partir dos problemas propostos, o presente trabalho tem como objetivo identificar quais são as possíveis falhas nos projetos. Além de responder as perguntas em questão, o presente trabalho busca também avaliar e propor ações que mitiguem os riscos. Além disso, a pesquisa também verificará as causas e efeitos das falhas e dos riscos, assim por meio das ações propostas, espera-se que as falhas nos projetos sejam reduzidas. Para que as perguntas do problema proposto sejam respondidas e os objetivos sejam alcançados, serão utilizadas técnicas de gerenciamento de risco a fim de auxiliar na identificação, avaliação e mitigação dos riscos nos projetos de consultoria.

Para a realização da pesquisa foi elaborada uma revisão bibliográfica com os seguintes temas abordados: riscos, gerenciamento de riscos e gerenciamento de riscos em projetos; após a revisão bibliográfica, são apresentadas algumas técnicas de gerenciamento de riscos; depois a apresentação das técnicas de gerenciamento de risco é apresentada a metodologia utilizada para a realização desta pesquisa, seguida dos resultados e comentários da pesquisa e finalizando com a conclusão.

\section{REVISÃO TEÓRICA}

\section{Riscos}

Raz et al. (2002) definem risco como uma condição indesejável que causa atraso, aumenta os custos e produz resultados desfavoráveis para os projetos, organizações, sociedade e ambientes. Segundo Andreta (2014), risco é uma medida da probabilidade de um efeito adverso sobre o objetivo de um processo. Segundo Ritchie et al. (2007), a palavra risco está associada a eventos que influenciam a eficiência/eficácia da 
continuidade do negócio. Segundo Salavati et al. (2016), o risco também está relacionado com o desempenho, ao controlar e reduzir o risco o desempenho será melhorado. Penha et al. (2014) explicam que, riscos são entendidos como eventos ou condições de incertezas que, se ocorrerem, terão efeitos negativos sobre ao menos um objetivo do projeto.

Segundo Penha et al. (2014), fatores como gerenciamento deficiente, inexistência de métodos, falhas na comunicação, implantação de vários projetos simultaneamente, dependência de participantes externos e falta de qualificação de equipe são apontados como causas de riscos. Se essas causas não forem contornadas, pode haver um resultado negativo para a organização.

As organizações precisam identificar e gerenciar todos os aspectos de risco, considerando que riscos não identificados são algumas das maiores causas de fracasso de projetos (SALAVATI et al., 2016). Slavati et al. (2016) alertam que a identificação das necessidades e as exigências dos clientes podem conter alguns riscos. Assim, recomendam buscar o feedback de recursos externos, tais como os clientes. Segundo os autores, outro fator de contenção de riscos é o apoio de gestores que devem motivar os membros da equipe de um projeto para otimizar os processos.

Segundo Vesco et al. (2014), uma empresa está sempre sujeita a uma grande diversidade de riscos durante a condução de seus negócios e conhecê-los é fundamental. Alguns riscos são comuns a todas as organizações, outros são específicos às determinadas atividades ou setores, mas a identificação dos riscos, a capacidade de gerenciá-los, o conhecimento das probabilidades de ocorrência de cada risco e o impacto de sua materialização podem ser objetos de uma gestão baseada em riscos.

\section{Gerenciamento de Riscos}

Algumas pesquisas desenvolvidas servem para estruturar a gestão de risco como conhecimento científico. A gestão de risco envolve atividades de controle de gestão que atrelados, podem ser verificados por meio de estruturas padrões de gerenciamento de risco. Estas estruturas têm como objetivo descrever, definir e estabelecer critérios que possam ajudar a melhorar o controle de uma organização, permitindo dar suporte para alcançar uma maior eficiência organizacional, além de permite também, uma maior flexibilidade para enfrentar mudanças e eventos adversos (VESCO et al., 2014).

A norma ISO 31000 tem como finalidade padronizar a gestão de risco. Conforme explicado na norma, a validação de risco é um processo global que envolve basicamente 3 processos: identificar, analisar e avaliar os riscos. Como primeiro passo é preciso que a organização identifique as fontes de risco, suas causas e consequências potenciais, já o segundo passo é necessário que se analise os riscos identificados com a finalidade de desenvolver as suas compreensões. Após analisar os riscos identificados é preciso que se analise suas causas, fontes, consequências e probabilidades de ocorrência. O terceiro passo é auxiliar na tomada de decisão com base nos resultados da análise sobre quais riscos necessitam de tratamento e qual a prioridade.

Oliveira et al. (2017) analisaram a possibilidade de aplicar a norma ISO 31000 como um processo sistemático de Gerenciamento de Risco da Cadeia de Suprimentos (GRCS). Para isso os autores realizaram uma revisão bibliográfica em artigos publicados de 2004 a 2015 nas bases de dados mais relevantes (Web of 
Science e Scopus). O objetivo foi analisar a correspondência das etapas utilizadas por diferentes autores na literatura com as etapas do processo de gestão de riscos proposto no ponto 5.4 da norma que cita três processos: identificar, analisar e avaliar. Este tópico não chega a falar do tratamento dos riscos e implementação de ações. Os pesquisadores encontraram um consenso na literatura, embora alguns autores defendam menos ou mais passos para a sua realização. Desconsiderando diferentes variações nos nomes dados a cada uma das etapas, o consenso obtido foi: mais de $90 \%$ dos trabalhos analisados utilizam a etapa de avaliação de riscos; mais de $80 \%$ passaram pelo processo de identificação dos riscos; o terceiro passo mais utilizado com mais de $60 \%$ foi o a mitigação de riscos e o quarto mais utilizado foi a etapa de monitoramento de risco. Assim, os autores afirmam que $100 \%$ dos artigos analisado podem ser cobertos pela norma ISO 31000

Glória Júnior (2015) constatou em sua pesquisa que a identificação dos riscos e a resposta aos riscos são temas recorrentes a todas as abordagens. Os participantes das atividades de identificação podem incluir: o gerente do projeto, membros da equipe do projeto, clientes, especialistas no assunto que são externos à equipe, usuários finais, outros gerentes de projetos, partes interessadas e especialistas em gerenciamento de riscos. Embora essas pessoas sejam os principais participantes da identificação dos riscos, todos envolvidos no projeto devem ser estimulados a realizar o processo de identificação. Com relação a resposta aos riscos, o autor explica que esta envolve a escolha de alternativas estratégicas, ações corretivas e modificações nos planos dos projetos.

Segundo Sádaba et al. (2013), a gestão de riscos é um processo sistemático que visa identificar, analisar, avaliar e gerenciar riscos, a fim de agir sobre a sua aparição (eliminando, mitigando ou controlando), por sistemas e procedimentos de execução para tratar os riscos inerentes a qualquer projeto. Os métodos no processo de gestão de riscos são integrados de acordo com o procedimento de trabalho onde, a partir de uma causa de falha, são identificados seus efeitos. Segundo os autores, a gestão de riscos em cada fase do projeto melhora as taxas de sucesso do projeto. Uma vez que se decide implementar um projeto de gestão de risco, a próxima fase do projeto é o planejamento, que inclui: i) definir a frequência e os momentos para monitorar indicadores e as pessoas responsáveis por isso; ii) detectar a maioria dos problemas que podem surgir durante o projeto (origem, consequências, avaliação, probabilidade, gravidade, plano de resposta e pessoa responsável); e iii) atualização periódica. Após a análise e avaliação de risco, o gerente de projeto deve estabelecer planos de ações com base nas recomendações para cada nível de risco, e priorizar as ações mais importantes. A implementação de ações corretivas deve ser imediata, ajustando orçamentos, identificando obstáculos, verificando recursos, analisando tarefas anteriores, tarefas atrasadas e tarefas não planejadas, assim como corrigindo os resultados inadequados.

\section{Riscos em Projetos}

Segundo Mulisani et al. (2014), um projeto é um esforço temporário usado para criar algo que não tenha sido feito antes e que não vai ser feito novamente no futuro. Quanto maior a novidade de um produto ou serviço, menos claras serão as informações iniciais, o que garante um risco maior para o projeto. Devido 
a esta singularidade, convive-se com o risco e a incerteza de se fazer algo novo e dependendo da fase do projeto os riscos implicarão em maior ou menor influência no resultado. 0 gerenciamento de risco permite a organização identificar os riscos existentes em seus projetos, possibilitando o desenvolvimento de ações para controlá-los.

Raz et al. (2002) definiram os riscos em projetos como eventos indesejáveis que podem causar atrasos, gastos excessivos, resultados insatisfatórios, riscos à segurança ou riscos ambientais, e até mesmo o fracasso total do projeto. Segundo os autores, qualquer gerente de projeto sabe que não há projeto livre de riscos, cada projeto é diferente, e envolve um certo grau de incerteza, mas, muitas organizações tendem a assumir que todos os seus projetos terão sucesso, e acabam deixando de analisar os riscos. Quando uma organização deixa de analisar esses riscos, ela acaba não se preparando para os erros que possam vir a ocorrer. Por não estarem preparadas para esses erros, as organizações acabam não atingindo os resultados esperados em seus projetos.

Segundo Sádaba et al. (2013), os projetos são os principais métodos para a mudança em uma empresa. Para minimizar as possíveis consequências negativas é necessário ter metodologias apropriadas. Mesmo a metodologia de risco mais simples, se aceita pela organização e aplicada corretamente, pode aumentar as chances de sucesso. $O$ objetivo final de qualquer sistema de gerenciamento de risco em projeto é aumentar a probabilidade de sucesso nos projetos. Sádaba et al. (2013) concluíram sua pesquisa afirmando que os gerentes de projeto que utilizaram a metodologia de gerenciamento de risco relataram menos problemas surgidos em seus projetos, e que o tempo gasto na aplicação do método vale a pena os resultados.

Segundo Penha et al. (2014), os possíveis fracassos de um projeto, muitas das vezes, se encontram nos objetivos estabelecidos, nas metas mal planejadas, na falta ou inconsistência de informações, na falta de detalhamento de suas atividades e nos erros de estimativa e a alocação ineficiente de recursos empregados. Esses são alguns fatores de risco que podem impactar diretamente na entrega do projeto ou até mesmo causar o cancelamento do mesmo. Esses problemas acontecem devido à falta de conhecimento do risco dos processos envolvidos no projeto.

\section{Técnicas de Gerenciamento de Riscos}

Guebitz et al. (2012) propõem métodos de análise de risco, como Análise de Modos de Falha e Efeitos (FMEA), Análise De Efeitos de Modos de Falha e Criticidade (FMECA) ou uma Análise de Perigos e Pontos Críticos de Controle (HACCP), além dos métodos de facilitação de gestão de risco, como o Diagrama de Causa e Efeito. Oliveira et al. (2010) aplicaram processos de gerenciamento de risco no setor de prestação de serviço, que segundo os mesmos é um tema pouco explorado na literatura. Para realizar a aplicação de tais processos foi realizada uma pesquisa bibliográfica para conceituar e entender as técnicas utilizadas além de estudar e analisar a integração dessas técnicas e, por fim aplica-las. Os autores utilizam das seguintes técnicas: Análise de Processos Críticos por Especialistas (APCE); Análise da Árvore de Falhas (que é semelhante ao Diagrama de Causa e Efeito); e Análise de Modos de Falha e Efeitos (FMEA). 
Segundo Raz et al. (2002), os projetos diferem em muitos aspectos, tais como tamanho, duração, incertezas, complexidade, ritmo, objetivos, restrições e outras dimensões. Os autores concluíram que não se pode esperar que um único processo de gestão de risco universal e seu conjunto de técnicas seja aplicável a todos os tipos de projetos. Assim como existem diferentes tipos de projetos, deve-se esperar diferentes tipos de práticas de gestão de risco.

\section{Diagrama de Causa e Efeito (DCE)}

O DCE foi projetado para classificar e categorizar as potenciais causas de um problema ao organizar as relações causais. Uma desvantagem desta técnica é que ela é fortemente dependente do conhecimento detalhado do problema, porém, equipes que usam o diálogo e a discussão efetivamente em um contexto de apoio podem superar a essa restrição. A vantagem deste método é que quebrar as causas em mais detalhes ajuda a organizar e relacionar os fatores (DOGGETT, 2005).

Segundo Cruz et al. (2013), o DCE serve como taquigrafia visual para representar danos potenciais para a qualidade, segurança, eficácia e a relação desses possíveis danos uns aos outros. Trata-se de identificar potenciais variáveis que podem ter um impacto sobre o atributo desejado, pois a identificação de potenciais riscos em um DCE se destina a ser qualitativo, nenhuma quantificação da severidade ou probabilidade é dada a estes fatores de risco.

Trata-se de uma técnica simples que pode ajudar a estruturar um processo de análise de risco e comumente utilizada durante um FMEA como um método de facilitação para identificar as causas de um modo de falha, mas esta técnica pode ser usada para determinar de forma sistemática as causas e subcausas de um determinado evento. De forma ilustrada, o evento global ou o problema é indicado na extremidade direita de uma seta horizontal e causas e subcausas são determinados por meio do preenchimento da 'espinha de peixe' conforme ilustrado na Figura 1 (GUEBITZ et al., 2012).

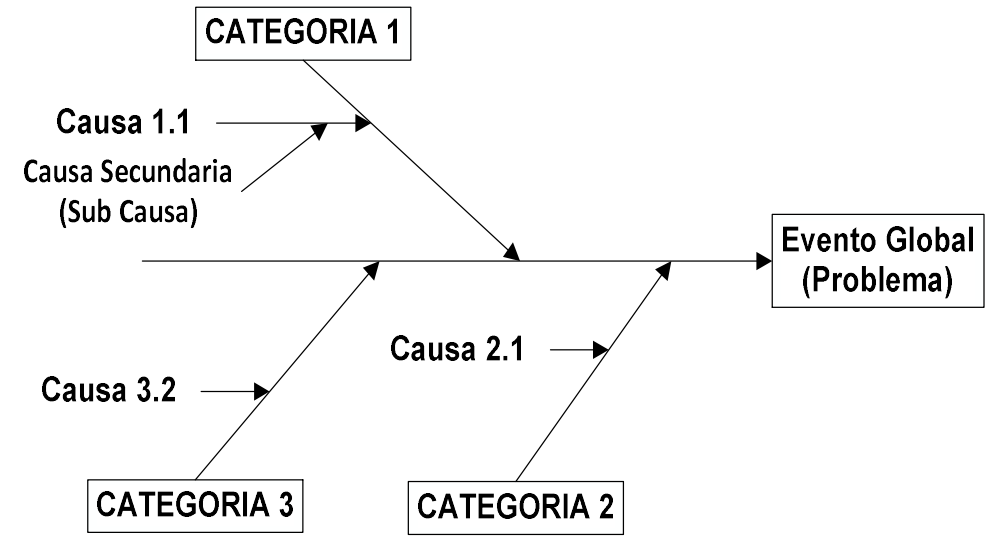

Figura 1: Exemplo de Diagrama de Causa e Efeito.

\section{Análise de Causa Raiz (ACR)}

A Análise de Causa Raiz (ACR) é um processo reativo, ocorre após o dano já ter sido feito, por essa razão, procura-se conhecer o conjunto causal de cada um de todos os efeitos possíveis utilizado. Para 
identificar e explicar os fatores básicos ou causais que sustentam a variação no desempenho e que podem produzir resultados adversos, que podem ser vistos como riscos (LATINO, 2009; SENDERS, 2004). A forma como a ACR é implementada pode variar entre as organizações de acordo com seus regimes regulatórios, cultura organizacional ou procedimentos internos. Não é uma técnica única e bem definida, mas sim uma abordagem geral para descobrir as causas de um evento e os fatores que contribuem para um incidente (CARD et al., 2012).

Conforme Doggett (2005), para se resolver um problema é preciso identificar a causa desse problema e tomar medidas para eliminar a causa, mas se a causa raiz de um problema não é identificada, então os sintomas e o problema continuarão a existir. Vários métodos existem para identificar a causa raiz, mas a eficácia depende da aplicação rigorosa do grupo e da integridade da causa raiz selecionada.

A prática de ACR é focada na crença de que os problemas são melhor resolvidos pela tentativa de corrigir ou eliminar a causa raiz, em oposição a meramente abordar os sintomas. Ao direcionar as medidas corretivas na causa raiz, espera-se que a probabilidade de recorrência do problema será minimizada (REID et al., 2012). Segundo Rooney et al. (2004), a causa raiz é aquela sob a qual a gerência tem controle e pode gerar recomendações eficazes. Deve-se evitar o uso de classificações de causas gerais, tais como erro do operador, falha do equipamento ou fator externo.

Com relação as etapas para a realização da ACR, Rooney et al. (2004), Murugaiah et al. (2003), e Reid et al. (2012), citam 4 etapas: Coleta de Dados; Fatores Causais Gráficos, o qual é simplesmente um diagrama de sequência que descreve os eventos que levaram a uma ocorrência conforme ilustra a Figura 2; Identificação da Causa Raiz; Geração e implementação de recomendações.

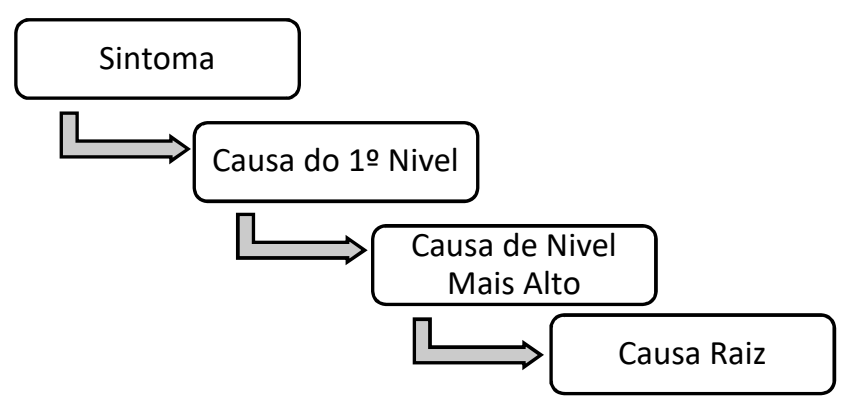

Figura 2: Diagrama de Fator Causal. Fonte: Adaptação de ACR, Rooney et al. (2004), Murugaiah et al. (2003) e Reid et al. (2012).

Segundo Pham et al. (2010), embora a ACR seja eficaz na identificação de perigos, muitas vezes não oferece métodos para mitigar os riscos. Sendo assim, indicam a utilização de mais métodos juntos a ACR. Segundo Murugaiah et al. (2003), uma técnica normalmente utilizada para a estruturação da ACR é o DCE, mas além deste, foi utilizado também o método dos '5 Porquês'. Segundo Ayad (2010), perguntar 'por quê' cinco vezes consecutivas é uma abordagem que muitos profissionais de Seis Sigma usam para ajudar a identificar a causa raiz de um problema. Guerin (2015), também utilizou a ACR baseado no DCE afim de verificar os fatores que contribuem para um determinado evento. Segundo Doggett (2005), o DCE é uma técnica fácil de usar para desenvolver e classificar categorias de causa raiz. Segundo Reid et al. (2012), na 
maioria das vezes, os praticantes tendem a usar brainstorming, FMEA ou '5 Porquês' para determinar a causa raiz. Reid et al. (2012), organizaram essas técnicas em um quadro, conforme demonstra o Quadro 1.

Quadro 1: Técnicas de ACR.

\begin{tabular}{|l|l|l|}
\hline TECNICA & IDENTIFICAÇÃO DA CAUSA RAIZ & CENÁRIO \\
\hline FMEA & $\begin{array}{l}\text { - Priorização de risco: os modos de falha e os efeitos são dados escores para } \\
\text { determinar a magnitude do risco } \\
\text { - Análise de causa: Permite o redesenho do sistema de processos de alta prioridade }\end{array}$ & Circunstâncias de falha \\
\hline $\begin{array}{l}\text { 5 Por } \\
\text { quês }\end{array}$ & $\begin{array}{l}\text { - Por que um problema ocorre é solicitado cinco vezes para chegar ao núcleo de um } \\
\text { problema }\end{array}$ & $\begin{array}{l}\text { Problemas ou defeitos do } \\
\text { processo }\end{array}$ \\
\hline DCE & - Uma técnica de análise usada para examinar o desempenho humano & Análise de causa e efeito \\
\hline
\end{tabular}

Fonte: Reid et al. (2012).

\section{Porquês}

A técnica ' 5 Porquês' sugere que, perguntando repetidamente 'Por quê?, é possível 'descascar' as camadas de sintomas que levam à causa raiz de um problema, embora chamada de ' 5 Porquês', pode-se fazer a pergunta menos ou mais do que cinco vezes antes que a questão relacionada ao problema seja descoberta (LATINO, 2009). Tal método envolve olhar para qualquer problema e perguntar: 'Por quê?' e ‘O que causou este problema?'. A análise '5 Porquês' da Figura 3 começa com a típica questão de porquê determinado evento ocorre. Este evento pode ser oriundo de dois outros eventos (risco A e B), onde pode haver mais de uma resposta (motivos) para cada pergunta. A aplicação da análise dos ' 5 Porquês' fornece uma abordagem baseada em fatos para a identificação e correção de problemas que se concentram não apenas na redução de defeitos, mas também na sua eliminação (MURUGAIAH et al., 2010).

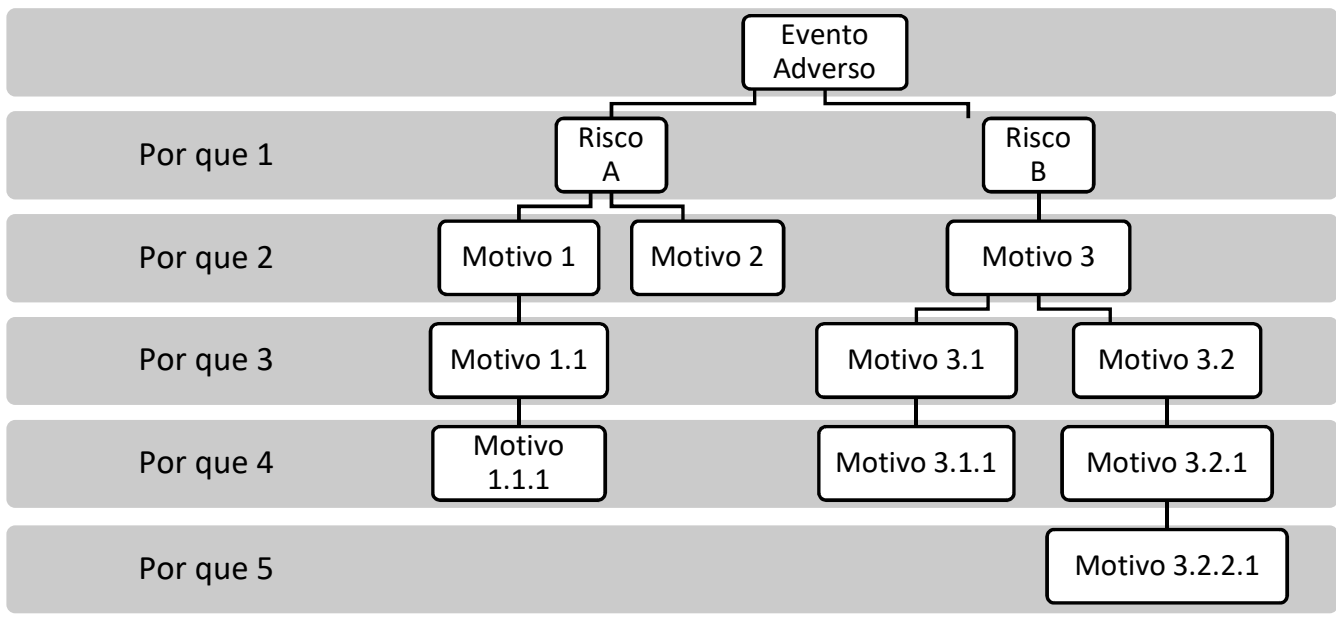

Figura 3: Análise '5 Porquês'. Fonte: Adaptado de Murugaiah et al. (2010).

Murugaiah et al. (2010), concluíram em sua pesquisa que soluções capazes de eliminar em vez de reduzir as falhas podem ser descobertas e implementadas. Concluíram também que soluções de baixo custo ou custo zero poderiam ser implementadas para eliminar desperdícios ou defeitos. Segundo Rooney et al. (2004), é necessário aprofundar no evento ocorrido, questionando se os envolvidos no evento estavam confusos ou familiarizados com as tarefas que resultaram no evento. Segundo Ayad (2010), a qualidade e a precisão dos '5 Porquês' depende de pensamento crítico. Os participantes são convidados a refletir sobre a operação, considerando o contexto da situação. A falta de pensamento crítico daqueles que foram 
perguntados pode levar a respostas erradas e conclusões erradas. Limitar o questionamento a 'por que' em qualquer situação priva o pesquisador de uma riqueza de informações que podem ser adquiridas fazendo mais perguntas, como por exemplo: Quais são as razões? Há alguma falácia no raciocínio? Quão boa é a evidência?. O trabalho de Ayad (2010) demonstrou que a metodologia do '5 Porquês' para identificar causas de defeitos é insuficiente sem o pensamento crítico, pois com ele tem a capacidade de explorar o contexto da situação e fornece uma ampla plataforma para a compreensão de padrões, consequências e riscos.

Segundo Guerin (2015) o método de ACR pode ser comparado com o método dos '5 Porquês'. Uma vez questionado o 'porquê' de uma resposta, isso produz outras razões para a(s) causa(s) de um incidente. Assim, o método dos '5 Porquês' utilizado no trabalho de Guerin (2015) auxiliou a uma análise mais profunda do ACR. A vantagem do método '5 Porquês' é que ele pode ser feito usando recursos limitados (geralmente um indivíduo ou uma pequena equipe). Porém, depende de julgamento profissional para garantir a linha de questionamento do evento sob investigação. A comparação qualitativa desses dois métodos é feita no Quadro 2.

Quadro 2: Comparação Qualitativa da ACR e o método de '5 Porquês'.

\begin{tabular}{|l|l|}
\hline ACR & 5 Porquês \\
\hline Detalhada e demorada & De alto nível, o rápido \\
\hline $\begin{array}{l}\text { Requer treinamento intensivo de pessoal para conduzir a } \\
\text { pesquisa (vários dias) }\end{array}$ & Curta duração (de uma a duas horas) \\
\hline $\begin{array}{l}\text { Minuciosa, ampla colaboração na tomada de decisões, } \\
\text { envolvendo uma ampla gama de especialistas de diversas } \\
\text { origens }\end{array}$ & $\begin{array}{l}\text { Breve, com um mínimo de consultas / colaboração para decidir sobre } \\
\text { as causas, concebido para ser conduzido por indivíduos ou pequenas } \\
\text { equipes }\end{array}$ \\
\hline $\begin{array}{l}\text { Requer nível significativo de recursos (requer uma } \\
\text { equipe de pelo menos 5-6) }\end{array}$ & $\begin{array}{l}\text { Pode ser feito usando recursos limitados (pode ser feito por um } \\
\text { indivíduo ou pequena equipe) }\end{array}$ \\
\hline $\begin{array}{l}\text { Fornece uma análise robusta das causas e fatores } \\
\text { contribuintes }\end{array}$ & $\begin{array}{l}\text { Depende de julgamento profissional para garantir a linha de } \\
\text { questionamento }\end{array}$ \\
\hline $\begin{array}{l}\text { Os resultados podem ser invocados para mudar de } \\
\text { processos de negócios ou de sistemas }\end{array}$ & $\begin{array}{l}\text { Dependência limitada pode colocar os resultados de uma única } \\
\text { análise }\end{array}$ \\
\hline $\begin{array}{l}\text { É aplicada em contextos comerciais onde uma lesão } \\
\text { significativa, morte ou incidente grave ambiental }\end{array}$ & $\begin{array}{l}\text { É aplicada em contextos comerciais como um método comum para a } \\
\text { análise de causa raiz, onde é necessária uma investigação de } \\
\text { incidentes }\end{array}$ \\
\hline
\end{tabular}

Autor: Guerin (2015).

\section{Análise de Modos de Falha e Efeitos (FMEA)}

A Análise de Modo de Falha e Efeitos (FMEA) é reconhecida como uma das técnicas mais benéficas nos programas de confiabilidade (ABDELGAWAD et al., 2010). Trata-se de um método utilizado na análise e avaliação de risco (SÁDABA et al., 2013). É um processo proativo destinado a prever os resultados adversos de várias falhas humanas e máquinas, e estados do sistema (SENDERS, 2005)

Segundo Sádaba et al. (2013), este método é um documento que contém quase toda a informação em um espaço pequeno. $\mathrm{O}$ documento contém a lista de riscos identificados e priorizados com base em índices de gravidade, detecção e de probabilidade, o que dará o Índice de Prioridade de Risco (IPR). O FMEA prioriza os riscos de acordo com o seu impacto potencial, a fim de desenvolver ações.

O FMEA é uma técnica estruturada que pode ajudar na identificação de modos de falhas, suas causas e efeito dentro de um sistema, além de planejar ações corretivas, assim dentro do contexto, o grau de criticidade de um modo de falha é determinado calculando o Índice de Prioridade de Risco (IPR) que é 
calculado a partir dos termos de gravidade, ocorrência, e de detecção de um modo de falha. Os componentes do sistema que são avaliados com um alto IPR são assumidos como os mais críticos. A gravidade (severidade) é utilizada para representar os efeitos potenciais associados com a ocorrência de um modo de falha, sendo que a ocorrência é a frequência da falha, além que a detecção é a dificuldade de detectar o risco. Cada um desses três termos recebe uma escala numérica que varia entre 1 e 10, sendo o resultado, vindo da multiplicação entre esses termos, será o IPR (PUENTE, 2006; ABDELGAWAD et al., 2010).

Segundo Puente (2006), é importante que o FMEA comece com um diagrama de fluxo que defina claramente as atividades ou processos a serem analisados. Técnicas como o DCE podem ser utilizados para determinar a relação entre a falha, efeitos e causas. Segundo Senders (2004), a utilização do FMEA junto com o ACR melhorará a segurança, pois é o espelho temporal da ACR, já que, enquanto o FMEA aguarda no tempo o ACR olha para o que já ocorreu.

\section{Brainstorming}

Lyons et al. (2004) realizaram uma pesquisa sobre o uso das técnicas de gestão de risco e evidenciaram que as técnicas mais utilizadas para identificar riscos são: Brainstorming, abordagem a qual é baseada em Casos e Listas de Verificação. Raz et al. (2001) realizaram um estudo com o objetivo de identificar quais os métodos mais utilizados e também quais os métodos que estão associados ao sucesso da gestão de projetos em geral e, em particular, à gestão de riscos do projeto. Os 38 métodos analisados pelos autores foram divididos em 6 grupos de acordo com a finalidade de cada técnica. $O$ grupo que continha os métodos de identificação de riscos contou com 4 métodos: Brainstorming, Formulário de Documentação de Risco, Listas de Verificação e Relatórios Periódicos de Risco. Os resultados da pesquisa constataram que o brainstorming é a técnica de identificação de risco mais comum utilizada e de maior sucesso.

Nas pesquisas de Cicek et al. (2013), Zhao et al. (2012), Vázquez-Valencia et al. (2016) e Krehbiel et al. (2009) foi utilizado o brainstorming juntamente com o FMEA. Os modos de falha potenciais foram determinados por meio de brainstorming, além do mais, na pesquisa de Krehbiel et al. (2009), a sessão de brainstorming resultou na criação do DCE, onde para cada causa geral, a equipe de pesquisa perguntou 'Por quê?', até que a causa raiz do problema fosse identificada. Conforme Puente (2006), o método FMEA baseiase em uma sessão de brainstorming destinada a descobrir as falhas que possam ocorrer em um sistema ou processo.

\section{METODOLOGIA}

Após o levantamento dos conceitos de gerenciamento de riscos em projetos e o levantamento de algumas das técnicas de gerenciamento de risco, a terceira fase da pesquisa trata-se da pesquisa de campo na empresa selecionada. Conforme visto na revisão da literatura deve-se esperar diferentes tipos de práticas de gestão de risco para diferentes projetos, além do mais, muitas das técnicas são utilizadas em conjunto para uma melhor análise. Sendo assim, após a revisão da literatura sobre gerenciamento riscos e suas técnicas, a presente pesquisa contará com 7 passos para alcançar os objetivos: Definição de um plano de 
gestão de riscos; Identificação dos riscos operacionais (Brainstorming); Análise da Causa Raiz (ACR) de cada falha; Estruturação da ACR de modo visual (DCE); Quantificação dos modos de falhas por meio do FMEA; Definição da função com maior Índice de Prioridade de Risco (IPR); e Recomendações de ações corretivas.

Após a definição de um plano de gestão de riscos estabelecendo as técnicas que serão utilizadas, iniciou-se a pesquisa em campo. Os métodos descritos acima bem como seus respectivos passos estão associados na Figura 4. No primeiro passo da pesquisa foi realizado um brainstorming com a finalidade de identificar as possíveis falhas nos projetos de consultoria da empresa. O processo foi realizado em um único momento com todos participantes ao mesmo tempo, sendo assim, neste processo tiveram 3 participantes: os dois sócios gestores da organização e o funcionário de maior tempo de trabalho na organização (5 anos).

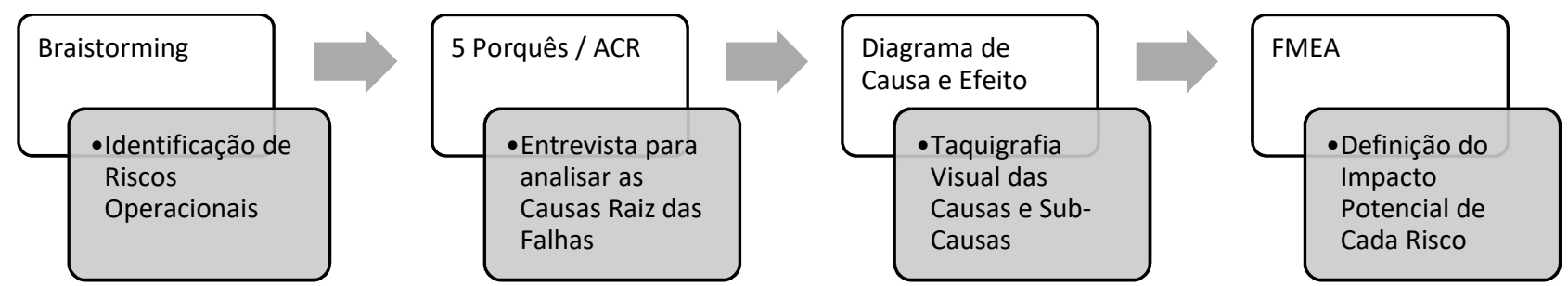

Figura 4: Métodos utilizados e etapas da pesquisa.

Na pesquisa de Ayad (2010), foi evidenciado a importância de pensamento crítico no método '5 Porquês'. Por esse motivo os envolvidos na pesquisa são os funcionários mais conceituados e com mais tempo de empresa. Conforme mencionado pelos autores Guerin (2015) e Murugaiah et al. (2003), o método dos ' 5 Porquês' pode ser utilizado para chegar-se a uma causa raiz de um problema, por se tratar de um método rápido e que pode ser aplicado a um número reduzido de participantes, foi julgado como um método ideal para o presente trabalho.

O objetivo nesta segunda fase foi de identificar a causa raiz de cada uma das falhas identificadas no processo de brainstomring. Durante a segunda fase, os participantes foram questionados de forma a explicar por qual motivo (ou por quais motivos) aquela falha ocorria. Dessa forma a técnica ' 5 Porquês' auxiliou a identificar a causa raiz de cada falha citada.

Com a identificação das falhas mais profundas e suas respectivas origens foi possível estruturar e visualizar o caso por meio da elaboração do DCE. Conforme os autores Guebitz et al. (2012), Latino (2009) e Cruz et al. (2013), o método é utilizado para determinar de forma sistemática causas e subcausas de um evento e possibilita olhar para os efeitos das causas que contribuem para um efeito de forma sistemática. Tendo identificado as causas de falhas e suas causas raízes nos projetos de consultoria, o próximo passo foi quantificar estas causas por meio do método FMEA que conforme Abdelgawad et al. (2010) ajuda a priorizar as falhas através de índices estabelecidos.

\section{RESULTADOS}

Através da realização do brainstorming no primeiro passo da pesquisa, foi possível obter uma lista de riscos associadas aos projetos de consultoria. Porém, esta lista não estava estruturada para saber de onde eram provenientes os erros, sendo assim, no segundo passo foi realizada a ACR para cada falha identificada. 
Nesta etapa foi questionado o 'por que' e 'por quais motivos' de cada falha ocorrer. Baseado no diagrama apresentado por Murugaiah et al. (2010), o método ACR foi estruturado na Figura 5.

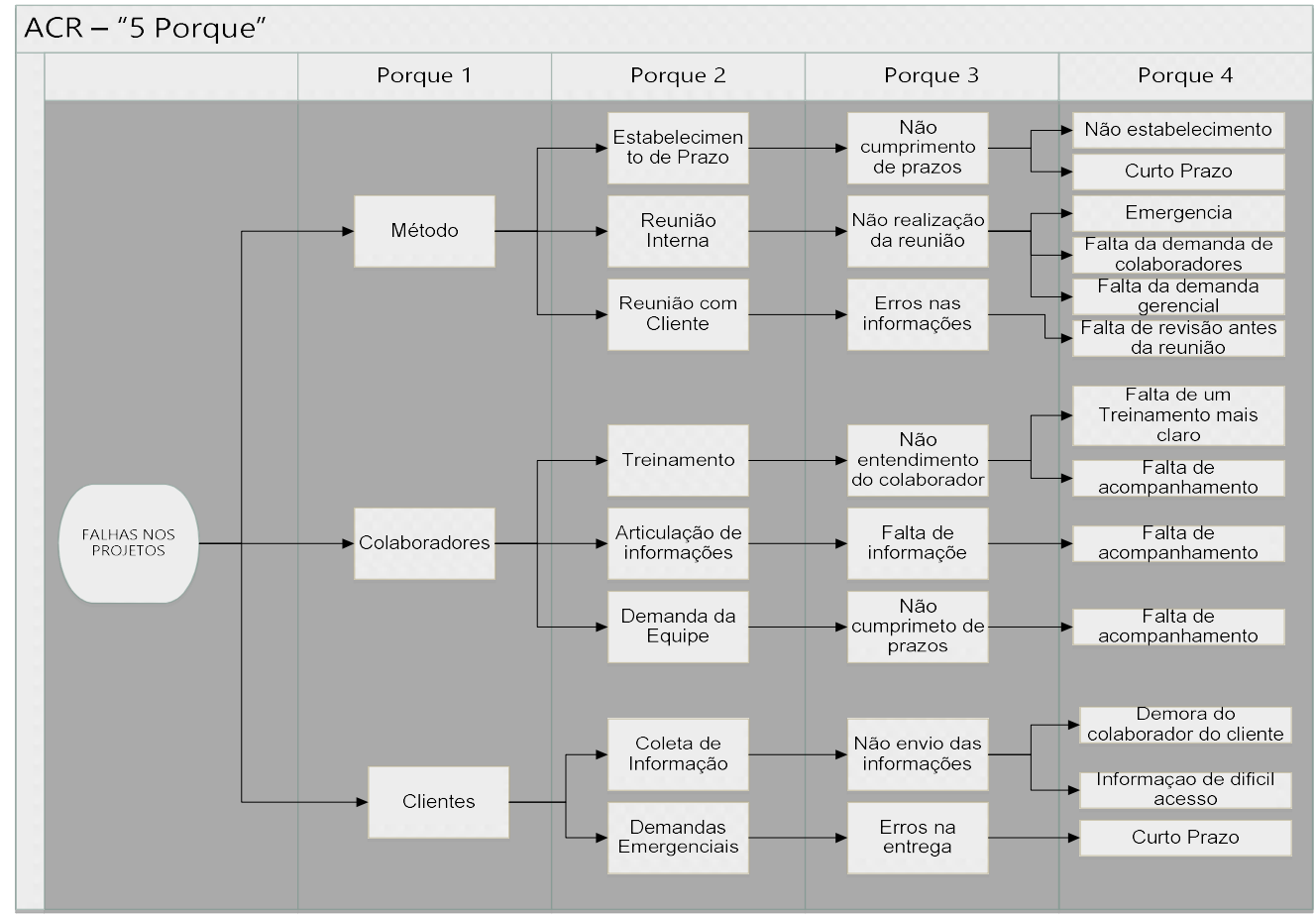

Figura 5: Diagrama de ACR com a metodologia '5 Porquês'.

Conforme Rooney et al. (2004), a ACR deve levar em consideração as causas que estão dentro da possibilidade de interferência da gerência. Sendo assim, as causas relacionadas aos clientes foram eliminadas da análise tendo em vista que a gerência não possui interferência nessas variáveis. As informações da ACR serviram de base para a criação do DCE que pode ser visto na Figura 6.

A elaboração do FMEA contou com a participação de todos participantes em mais uma sessão de brainstorming para identificar os modos e efeitos de falha. Assim também foi feito na pesquisa de Krehbiel et al. (2009), onde os modos de falha foram determinados através de brainstorming. Os modos e efeitos de falha podem ser visualizados no Quadro 4.

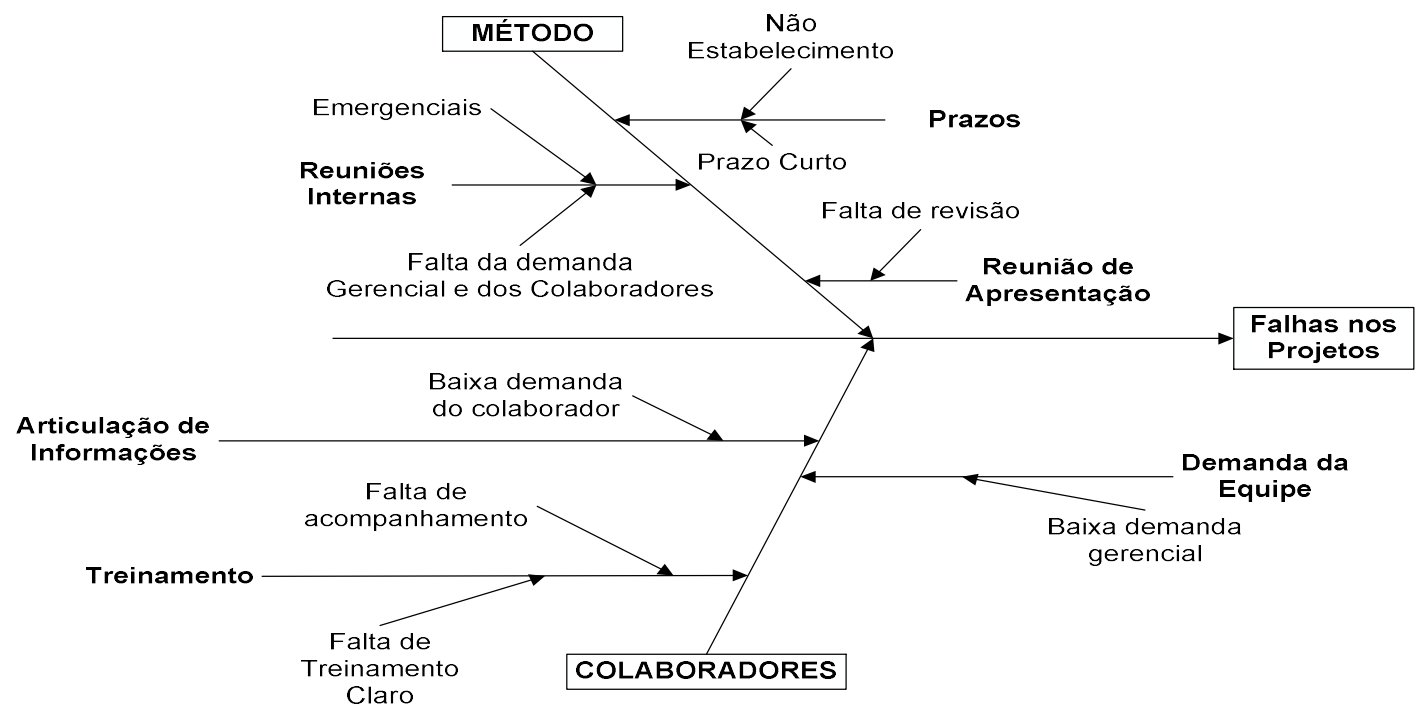

Figura 6: Diagrama de Causa e Efeito do Caso. 
Quadro 4: Modos e Efeitos de Falha.

\begin{tabular}{|c|c|c|c|}
\hline & Função do processo & Modo de Falha & Efeito(s) \\
\hline \multirow{3}{*}{ 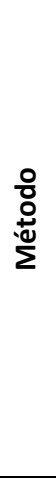 } & Estabelecimento de Prazos & $\begin{array}{l}\text { Não cumprimento de prazos do } \\
\text { projeto }\end{array}$ & $\begin{array}{l}\text { Comprometimento do Projeto; } \\
\text { Projeto não atendido. }\end{array}$ \\
\hline & $\begin{array}{l}\text { Reunião Interna (Controle e } \\
\text { Revisão) }\end{array}$ & Não realização da reunião & $\begin{array}{l}\text { Não estabelecimento de prioridades e } \\
\text { importâncias; } \\
\text { Não acompanhamento dos projetos; } \\
\text { Não realização da análise das informações } \\
\text { coletadas; } \\
\text { Retrabalho. }\end{array}$ \\
\hline & Reunião de Apresentação & Erros nas informações & $\begin{array}{l}\text { Erros na apresentação; } \\
\text { Retrabalho; } \\
\text { Comprometimento do Projeto. }\end{array}$ \\
\hline \multirow{3}{*}{$\begin{array}{l}\text { ஸे } \\
\frac{0}{0} \\
\frac{0}{0} \\
\frac{0}{0} \\
\end{array}$} & Treinamento & Não entendimento do colaborador & $\begin{array}{l}\text { Erros nas informações dos projetos; } \\
\text { Erros na apresentação; } \\
\text { Retrabalho. }\end{array}$ \\
\hline & Articulação de Informações & Falta de informações & Não cumprimento dos prazos do projeto. \\
\hline & Demanda da Equipe & Não cumprimento de prazos & Não cumprimento dos prazos do projeto. \\
\hline
\end{tabular}

É interessante citar que no último passo, que se tratava da criação do FMEA, no momento de estipular os modos e efeitos de falhas, notou-se algumas inconsistências nos processos de ACR, pois fez com que os participantes mudassem algumas opiniões com relação a algumas causas, sendo necessária uma segunda análise no método e também reconstruir o DCE, assim as etapas do processo de gerenciamento de risco foram refeitas conforme ilustrado na Figura 6.

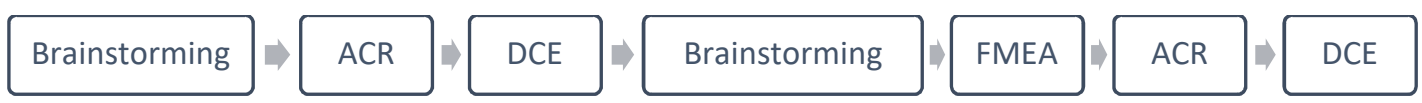

Figura 6: Métodos revisados e utilizados pelo autor.

Após estabelecidos os modos e efeitos das falhas e a retratação do DCE e da ACR, foi realizado o estabelecimento dos índices de Severidade, Ocorrência e Detecção. O resultado desta parte da pesquisa pode ser visto no Quadro 5. Com relação à coluna que diz respeito aos controles atuais dos processos, esta não foi inclusa, pois não há nenhum controle interno para nenhuma das funções.

Quadro 5: Elaboração do FMEA para projetos de consultoria.

\begin{tabular}{|c|c|c|c|c|c|c|c|c|c|}
\hline & & Possíveis Falha & & & & ICE & & & \\
\hline & $\begin{array}{l}\text { Função do } \\
\text { processo }\end{array}$ & $\begin{array}{l}\text { Modo de } \\
\text { Falha }\end{array}$ & Efeito(s) & Causa & 0 & $\mathbf{S}$ & D & $\begin{array}{l}\mathbf{N} \\
\mathbf{P} \\
\mathbf{R}\end{array}$ & Ação corretiva \\
\hline \multirow{5}{*}{ 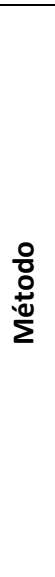 } & \multirow{2}{*}{$\begin{array}{l}\text { Estabelecimento } \\
\text { de Prazo }\end{array}$} & \multirow{2}{*}{$\begin{array}{l}\text { Não } \\
\text { cumprimento } \\
\text { de prazos }\end{array}$} & \multirow{2}{*}{$\begin{array}{l}\text { Comprometimento } \\
\text { do Projeto. } \\
\text { Cliente não } \\
\text { atendido. }\end{array}$} & $\begin{array}{l}\text { Não } \\
\text { estabelecimento }\end{array}$ & 4 & 8 & 3 & 96 & \multirow{2}{*}{$\begin{array}{l}\text { Sempre estabelecer } \\
\text { prazos. Verificar nível } \\
\text { de tarefas dos } \\
\text { colaboradores. }\end{array}$} \\
\hline & & & & Curto prazo & 8 & 7 & 5 & 280 & \\
\hline & \multirow[b]{3}{*}{$\begin{array}{l}\text { Reunião Interna } \\
\text { (Controle e } \\
\text { Revisão) }\end{array}$} & \multirow[b]{3}{*}{$\begin{array}{l}\text { Não } \\
\text { realização da } \\
\text { reunião }\end{array}$} & \multirow{3}{*}{$\begin{array}{l}\text { Não } \\
\text { estabelecimento } \\
\text { de prioridades e } \\
\text { importâncias. } \\
\text { Não } \\
\text { acompanhamento } \\
\text { do cliente. } \\
\text { Não realização da } \\
\text { análise das } \\
\text { informações } \\
\text { coletadas. }\end{array}$} & Emergências & 8 & 5 & 8 & 320 & \multirow[b]{3}{*}{$\begin{array}{l}\text { Estabelecer data fixa } \\
\text { para reunião interna e } \\
\text { evitar que outros } \\
\text { eventos atrapalhem. }\end{array}$} \\
\hline & & & & $\begin{array}{l}\text { Falta da demanda } \\
\text { de colaboradores }\end{array}$ & 6 & 5 & 5 & 150 & \\
\hline & & & & $\begin{array}{l}\text { Falta da demanda } \\
\text { gerencial }\end{array}$ & 7 & 5 & 6 & 210 & \\
\hline
\end{tabular}




\begin{tabular}{|c|c|c|c|c|c|c|c|c|c|}
\hline & $\begin{array}{l}\text { Reunião de } \\
\text { Apresentação }\end{array}$ & $\begin{array}{l}\text { Erros nas } \\
\text { informações }\end{array}$ & $\begin{array}{l}\text { Erros na } \\
\text { apresentação. } \\
\text { Comprometimento } \\
\text { do Projeto. }\end{array}$ & $\begin{array}{l}\text { Falta de revisão } \\
\text { antes da reunião }\end{array}$ & 6 & 9 & 5 & 270 & $\begin{array}{l}\text { Verificar informações } \\
\text { antes de apresentar } \\
\text { para o cliente. }\end{array}$ \\
\hline \multirow{4}{*}{$\begin{array}{l}\text { d } \\
\frac{0}{0} \\
\frac{0}{0} \\
\frac{0}{0} \\
\frac{0}{0}\end{array}$} & \multirow[t]{2}{*}{ Treinamento } & \multirow{2}{*}{$\begin{array}{l}\text { Não } \\
\text { entendimento } \\
\text { do } \\
\text { colaborador }\end{array}$} & \multirow{2}{*}{$\begin{array}{ll}\text { Erros } & \text { nas } \\
\text { informações } & \text { dos } \\
\text { clientes. } & \end{array}$} & $\begin{array}{l}\text { Falta de um } \\
\text { treinamento mais } \\
\text { claro }\end{array}$ & 5 & 6 & 8 & 240 & \multirow{2}{*}{$\begin{array}{l}\text { Dedicar mais tempo e } \\
\text { acompanhamento } \\
\text { aos novos } \\
\text { colaboradores em } \\
\text { treinamento. }\end{array}$} \\
\hline & & & & $\begin{array}{l}\text { Falta de } \\
\text { acompanhamento }\end{array}$ & 6 & 6 & 7 & 252 & \\
\hline & $\begin{array}{l}\text { Articulação de } \\
\text { informações }\end{array}$ & $\begin{array}{l}\text { Falta de } \\
\text { informações }\end{array}$ & $\begin{array}{l}\text { Demora na entrega } \\
\text { de trabalhos. }\end{array}$ & $\begin{array}{l}\text { Baixa demanda do } \\
\text { colaborador }\end{array}$ & 5 & 4 & 8 & 160 & \multirow{2}{*}{$\begin{array}{l}\text { Intensificar cobrança } \\
\text { da gerencia. }\end{array}$} \\
\hline & $\begin{array}{l}\text { Demanda } \\
\text { Equipe }\end{array}$ & $\begin{array}{l}\text { Não } \\
\text { cumprimento } \\
\text { de prazos }\end{array}$ & $\begin{array}{l}\text { Demora na entrega } \\
\text { de trabalhos. }\end{array}$ & $\begin{array}{l}\text { Baixa demanda } \\
\text { gerencial }\end{array}$ & 6 & 4 & 9 & 216 & \\
\hline
\end{tabular}

\section{DISCUSSÃO}

\section{Discussão Dos Métodos Utilizados}

Conforme a fundamentação teórica sobre gestão de risco, diversos autores afirmaram que a gestão de risco conta com o uso diversificado de técnicas, podendo ser feito de maneira diferente de acordo com a necessidade do caso e em ambientes diversificados (MULISANI et al., 2014; SÁDABA et al., 2013; RAZ et al., 2002). O Quadro 6 mostra uma relação de autores e técnicas utilizadas. O quadro se limita apenas às técnicas utilizadas neste estudo.

Quadro 6: Técnicas Utilizadas em gerenciamentos de risco.

\begin{tabular}{|l|l|}
\hline $\begin{array}{l}\text { Técnicas Citadas } \\
\text { ou Utilizadas }\end{array}$ & Autores \\
\hline Brainstorming & $\begin{array}{l}\text { Raz et al. (2002) Reid et al. (2012), Lyons et al. (2004), Raz et al. (2001), Cicek et al. (2013), Zhao et al. (2012), } \\
\text { Vazquez-Valencia, et al. (2016) e Krehbiel et al. (2009). }\end{array}$ \\
\hline RCA & $\begin{array}{l}\text { Andreta (2014), Card et. al., (2012), Pham et. al. (2010), Latino (2009), Senders (2004), Doggett (2005), Reid } \\
\text { et al. (2012), Rooney et al. (2004), Murugaiah et al. (2003), Ayad (2010), Guerin (2015). }\end{array}$ \\
\hline DCE & $\begin{array}{l}\text { Guebitz et al. (2012), Ariful et al. (2006), Latino (2009), Doggett (2005), Cruz et al. (2013), Guerin (2015), } \\
\text { Krehbiel et al. (2009) }\end{array}$ \\
\hline '5 Porque' & $\begin{array}{l}\text { Ariful et al. (2006), Reid et al. (2012), Latino (2009), Rooney et al. (2004), Murugaiah et al. (2010), Ayad (2010), } \\
\text { Guerin (2015), Krehbiel et al. (2009). }\end{array}$ \\
\hline FMEA & $\begin{array}{l}\text { Andreta (2014), Guebitz et al. (2012), Ariful et al. (2006), Reid et al. (2012), Abdelgawad et al. (2010), Sádaba } \\
\text { et al., (2013), Senders (2004), Puente (2006), Kasai et al. (2016). }\end{array}$ \\
\hline
\end{tabular}

\section{Discussão dos resultados do estudo empírico}

Com base nas informações extraídas como resultado da metodologia aplicada foi possível averiguar as funções mais críticas no processo. Conforme citado anteriormente, o Passo 6 do estudo consistia em identificar a função com maior IPR, sendo que foi obtida somando-se o IPR de cada causa da função e o resultado deste cálculo pode ser visto no Quadro 7, em concordância com o FMEA, a função que tem prioridade de ser tratada é a 'Reunião Interna'. Conforme proposto no 7o e último passo, foram estabelecidas as recomendações das ações corretivas. Para a função com maior IPR foi recomendado a seguinte ação: 'Estabelecer data fixa para reunião interna e evitar que outros eventos interfiram', porém, foi estabelecida uma recomendação para cada causa:

Quadro 7: Funções e IPR.

\begin{tabular}{|l|l|}
\hline Função do processo & IPR \\
\hline Estabelecimento de Prazo & 376 \\
\hline
\end{tabular}




\begin{tabular}{|l|l|}
\hline Reunião Interna (Controle e Revisão) & 680 \\
\hline Reunião de Apresentação & 270 \\
\hline Treinamento & 492 \\
\hline Articulação de informações & 160 \\
\hline Demanda da Equipe & 216 \\
\hline
\end{tabular}

A falta da demanda gerencial: a gerência responsável ficará encarregada de realizar a reunião semanalmente e também de criar métodos de acompanhamento, para melhorar a eficiência dos projetos; em caso de emergências que precisam ser resolvidas urgentemente, a data da reunião fica agendada para o dia seguinte; falta da demanda de colaboradores, os colaboradores deverão apresentar semanalmente o andamento de seus projetos.

\section{CONCLUSÕES}

A fim de atender ao objetivo inicial deste artigo, foi realizado um estudo teórico embasado em: risco; gerenciamento de riscos; e gerenciamento de riscos em projetos. Após o estudo teórico foram mostradas as técnicas utilizadas em um gerenciamento de risco. A partir da fundamentação teórica foi possível definir e planejar um plano de gestão de riscos. O estudo de caso foi realizado em uma empresa de prestação de serviços no ramo de consultoria de negócios com a finalidade de identificar falhas e risco em seus projetos.

A adoção da integração das técnicas Brainstorming, ACR, '5 Porquês', DCE e FMEA, proporcionaram ao estudo os processos dentro da norma ISO 31000 (identificar, analisar e avaliar riscos). Pode-se concluir, por meio da aplicação prática, que a proposta pelo uso integrado das técnicas selecionadas é pertinente e viável. Pode-se afirmar que as perguntas colocadas pelo problema foram respondidas e os objetivos da pesquisa foram realizados: foram identificadas as falhas capazes de trazer consequências negativas para os projetos da empresa, estas falhas foram analisadas e avaliadas. Foi também identificado as causas de cada uma dessas falhas juntamente com uma ação de correção para cada uma.

Foi verificado que a gestão de risco está envolvida tanto em casos de projetos (GLÓRIA JÚNIOR, 2015; MULISANI et al., 2014; SALAVATI et al., 2016), empresas (ARIFUL et al., 2006; PENHA et al., 2014; SÁDABA et al., 2013) e indústrias (ABDELGAWAD et al., 2010; GUERIN, 2015) como em casos de saúde (PHAM et al., 2010; CARD et al., 2012; CRUZ et al., 2013). Assim, a aplicação combinada destas técnicas dentro de projetos no setor de prestação de serviço é conveniente como contribuição de cunho científico. Este artigo apresenta um modelo de características integrativas inovadoras e o aplica em processos de projetos, um caso pouco explorado tanto na prática quanto na pesquisa acadêmica em serviços.

Não foi estabelecido por nenhum autor citado nesse estudo a ordem em que as técnicas devem ser utilizadas. No caso estudado, notou-se que após a elaboração do FMEA foi verificado que era necessário retornar a ACR, assim, também foi necessário refazer o DCE. Uma limitação pode ser notada no método: 0 julgamento dos fatores pelos participantes são valores estipulados pelas suas intuições, o que pode ser diferente de pessoa para pessoa.

É importante lembrar que este artigo tratou até a parte de sugerir ações mitigadoras, cabe a equipe de trabalho da empresa implementar as ações e realizar o processo de gerenciamento de risco 
continuadamente, nesse sentido, recomenda-se a continuação deste estudo verificando o resultado das ações corretivas e uma segunda análise das mesmas funções.

\section{REFERÊNCIAS}

ABDELGAWAD, M.; FAYEK, A.. Risk Management in the Construction Industry Using Combined Fuzzy FMEA and Fuzzy AHP. Journal of Construction Engineering and Management, v.136, n.9, p.1028-1036, 2010. DOI: https://doi.org/10.3846/13923730.2015.1068847

ANDRETA, M.. Some Considerations on the Definition of Risk Based on Concepts of Systems Theory and Probability. Risk Analysis, v.34, n.7, p.1184-1195, 2014. DOI: http://doi.org/10.1111/risa.12092

ARIFUL, I.; TEDFORD, J. D.; HAEMMERLE, E.. Strategic Risk Management Approach for Small and Medium-Sized Manufacturing Enterprises (SMEs): A Theoretical Framework. Management of Innovation and Technology, v.2, p.694-698, 2006. DOI: http://doi.org/10.1109/ICMIT.2006.262309

AYAD, A.. Critical thinking and business process improvement. The Journal of Management Development, v.29, n.6, p.556-564, 2010. DOI:

http://doi.org/10.1108/02621711011046521

CARD, A. J.; WARD, J.; CLARKSON, P. J.. Successful risk assessment may not always lead to successful risk control: A systematic literature review of risk control after root cause analysis. Journal Of Healthcare Risk Management, v.31, n.3, p.6-12, 2012. DOI: http://doi.org/10.1002/ihrm.20090

CICEK, K.; CELIK, M.. Application of failure modes and effects analysis to main engine crankcase explosion failure on-board ship. Safety Science, v.51, n.1, p.6-10, 2013. DOI: http://doi.org/10.1016/j.ssci.2012.06.003

CRUZ, C. N.; TYNER, K. M.; VELAZQUEZ, L.; C. HYAMS, K.; JACOBS, A.; B. SHAW, A.; JIANG, W.; LIONBERGER, R.; HINDERLING, P.; KONG, Y.; BROWN, P. C.; GHOSH, T.; STRASINGER, C.; SUAREZ-SHARP, S.; HENRY, D.; UITERT, M V.; SADRIEH, N.; MOREFIELD, E.. CDER Risk Assessment Exercise to Evaluate Potential Risks from the Use of Nanomaterials in Drug Produc. The AAPS Journal, v.15, n.7, p.623-628, 2013.DOI: http://doi.org/10.1208/s12248-013$\underline{9466-6}$

DOGGETT, M.. Root Cause Analysis: A Framework for Tool Selection. Quality Management Journal, v.12 n.4, p.34-45, 2005. DOI:

https://doi.org/10.1080/10686967.2005.11919269

GLÓRIA JÚNIOR, I.. A Identificação e Mitigação de Riscos em Projetos de Desenvolvimento Rápidos de Jogos Digitais. Revista de Gestão e Projetos, v.6, n.1, p.79-94, 2015. DOI: http://dx.doi.org/10.5585/10.5585

GUEBITZ, B.; SCHNEDL, H.; KHINAST, J. G.. A risk management ontology for Quality-by-Design based on a new development approach according GAMP 5.0. Expert Systems with Applications, v.39, n.8, p.7291-7301, 2012. DOI: https://doi.org/10.1016/j.eswa.2012.01.089
GUERIN, T. F.. Understanding the Causes of Spills From the Supply and Handling of Chemicals at Resource Construction Sites: A Case Study. Remediation Journal, v.25, n.3, p.115145, 2015. DOI: https://doi.org/10.1002/rem.21436

KASAI, N.; FUJIMOTO, Y.; YAMASHITA, I.; NAGAOKA, H.. The qualitative risk assessment of an electrolytic hydrogen generation system. International Journal of Hydrogen Energy, v.41, n.30, p.1330-13314, 2016. DOI: https://doi.org/10.1016/j.ijhydene.2016.05.231

KREHBIEL, T. C.; EIGHME, J. E.; COTTELL, P. G.. Morgan Systems, Inc.: Application of Six Sigma to the finance function. Journal of Accounting Education, v.27, n.2, p.104123, 2009. DOI: https://doi.org/10.4236/ajor.2016.64026

LATINO, R. J.. Optimizing FMEA and RCA efforts in health care. Journal of Healthcare Risk Management, v.24, n.3, p.21-28, 2009. DOI:

https://doi.org/10.1002/jhrm.5600240305

LYONS, T.; SKITMORE, M.. Project risk management in the Queensland engineering construction industry: A survey. International Journal of Project Management, v.22, n.1, p.51-46, 2004. DOI: https://doi.org/10.1016/S02637863(03)00005-X

MULISANI, R. V.; GARCEZ, M. P.. A Influência dos Principais Fatores de Risco nas Diferentes Fases do Ciclo de Vida dos Projetos de Capital em uma Empresa de Mineração de Grande Porte. Revista de Gestão e Projetos - GeP, v.5, n.1, p.28-41, 2014. DOI: http://dx.doi.org/10.5585/10.5585

MURUGAIAH, U.; BENJAMIN, S. J.; MARATHAMUTHU M. S.; MUTHAIYAH, S.. Scrap loss reduction using the 5-whys analysis. International Journal of Quality \& Reliability Management, v.27, n.5, p.527-540, 2010. DOI: http://doi.org/10.1108/02656711011043517

OLIVEIRA, U. R.; PAIVA, E. J.; ALMEIDA, D. A.. Metodologia integrada para mapeamento de falhas: uma proposta de utilização conjunta do mapeamento de processos com as técnicas FTA, FMEA e a análise crítica de especialistas.

Produção, v.20, n.1, p.77-91, 2010. DOI:

http://dx.doi.org/10.1590/S0103-65132010005000004

OLIVEIRA, U. R.; MARINS, F. A. S.; ROCHA, H. M.; SALOMON, V. A. P.. The ISO 31000 standard in supply chain risk management. Journal of Cleaner Production, v.151, n.10, p.616-633, 2017. DOI: https://doi.org/10.1016/j.jclepro.2017.03.054

PENHA, R.; KNIESS, C. T.; BERGMAN, D. R.; BIANCOLINO C A.. Emprego de Técnicas de Gerenciamento de Riscos Técnicos em uma Empresa de Desenvolvimento de Softwares. Revista Gestão \& Tecnologia, v.14, n.1, p.151173, 2014. DOI: https://doi.org/10.20397/21776652/2014.v14i1.549 
PHAM, J. C.; KIM, G. R.; NATTERMAN, J. P.; COVER, R. M.; GOESCHEL, C. A.; WU, A. W.; PRONOVOST, P. J.. ReCASTing the RCA: An Improved Model for Performing Root Cause Analyses. American Journal of Medical Quality, v.25, n.3, p.186-191, 2010. DOI: http://doi.org/10.1177/1062860609359533

PUENTE, J.; PINO, R.; PRIORE, P.; FUENTE, D.. A decision support system for applying failure mode and effects analysis. International Journal of Quality \& Reliability Management, v.19, n.2, p.137-150, 2005. DOI: https://doi.org/10.1108/02656710210413480

RAZ, T.; MICHAEL, E.. Use and benefits of tools for project risk management. International Journal of Project Management, v.19, n.1, p.9-17, 2001. DOI: https://doi.org/10.1016/S0263-7863(99)00036-8

RAZ, T.; SHENHAR, A. J.; DVIR, D.. Risk management, project success, and technological uncertainty. R\&D Management, v.32, n.2, p.101-109, 2002. DOI: http://doi.org/10.1111/1467-9310.00243

REID, X.; SMYTH-RENSHAW J.. Exploring the Fundamentals of Root Cause Analysis: Are We Asking the Right Questions in Defining the Problem? Quality and Reliability Engineering International, v.28 n.5, p.535-545, 2012. DOI: http://doi.org/10.1002/qre.1435

RITCHIE, B.; BRINDLEY, C.. Supply chain risk management and performance. International Journal of Operations \& Production Management, v.27, n.3, p.303-322, 2007. DOI: https://doi.org/10.1108/01443570710725563

ROONEY, J.; HEUVEL, V.; LEE, N.. Root Cause Analysis for Beginners. Quality Progress, v.37, n.7, p.45-53, 2004.
SÁDABAA, S. M.; PÉREZ-EZCURDIAA A.; LAZCANOB A. M. E.; VILLANUEVA, P.. Project risk management methodology for small firms. International Journal of Project Management, v.32, n.2, p.327-340, 2014. DOI: https://doi.org/10.1016/j.ijproman.2013.05.009

SALAVATI, M.; TUYSERKANI, M.; MOUSAVI, S. A.; FALAHI, N.; ABDI, F.. Improving new product development performance by risk management. The Journal of Business \& Industrial Marketing, v.31, n.3, p 418-425, 2016. DOI: https://doi.org/10.1108/JBIM-04-2013-0090

SENDERS, J. W.. FMEA and RCA: FMEA and RCA: the mantras; of modern risk management. Quality \& Safety in Health Care, v.13, n.4, p.249-250, 2004. DOI: http://dx.doi.org/10.1136/qshc.2004.010868

VÁZQUEZ-VALENCIA, A.; SANTIAGO-SÁEZ, A.; PEREA-PÉREZ, B.; LABAJO-GONZÁLEZ E.; ALBARRÁN-JUAN, M. E.. Utility of Failure Mode and Effect Analysis to Improve Safety in Suctioning by Orotracheal Tube. Journal of Peri Anesthesia Nursing, v.32, n.1, p.28-37, 2016. DOI: http://dx.doi.org/10.1016/j.jopan.2015.03.014

VESCO, D. G. D.; FERNANDES F. C.; RONCON A.. Controles de gestão atrelados ao gerenciamento de risco: uma análise das produções científicas brasileiras sob a perspectiva de redes sociais. REDES- Revista hispana para el análisis de redes sociales, v.25, p.163-185, 2014. DOI: https://doi.org/10.5565/rev/redes.472

ZHAO, X.; ZHU, Y.. Research of fmea knowledge sharing method based on ontology and the application in manufacturing process. International Workshop on Database Technology and Applications, p.1-4, 2010. DOI: http://doi.org/10.1109/DBTA.2010.5658936

A CBPC - Companhia Brasileira de Produção Científica (CNPJ: 11.221.422/0001-03) detém os direitos materiais desta publicação. Os direitos referem-se à publicação do trabalho em qualquer parte do mundo, incluindo os direitos às renovaç̃̃es, expansões e disseminações da contribuiç̃o, bem como outros direitos subsidiários. Todos os trabalhos publicados eletronicamente poderão posteriormente ser publicados em coletâneas impressas sob coordenação da Sustenere Publishing, da Companhia Brasileira de Produção Científica e seus parceiros autorizados. Os (as) autores (as) preservam os direitos autorais, mas não têm permissão para a publicação da contribuição em outro meio, impresso ou digital, em português ou em tradução. 